2005 IEEE AP-S IEEE Int. Symp. and USNC/URSI National Radio Sc. Meeting on

Antennas and Propagation, Vol. 4B, pp: 135-138, Washington DC, July, 2005.

\title{
A Novel Time Reversal Method for Target Detection in Cluttered Media
}

\author{
Jian-Gang Zhu, Yi Jiang, Daniel D. Stancil, and José Moura \\ Department of Electrical and Computer Engineering, \\ Carnegie Mellon University, Pittsburgh, PA 15213
}

\section{Introduction}

A cluttered environment with highly scattering objects significantly enhances the difficulty for detecting targets using electromagnetic wave. In this paper, we present a novel time reversal method that nullifies the scattering objects to allow automatic focusing of the propagated electromagnetic wave on a target that is to be detected.

Time reversal method was first developed in acoustic wave propagation [1]. It has been demonstrated that in an environment where multi-scattering-path dominates, focusing of acoustic wave with super resolution can be achieved using time reversal method [1][2]. Recent experiments have shown that the super resolution focusing effect in acoustic wave propagation can also be achieved electromagnetic wave propagation [3][4].

Here, a novel time reversal method is presented for target detection in a cluttered medium. The idea is to use a time reversal concept to nullify the highly scattering environment while enable automatic focusing of the electromagnetic wave on the target. Numerical simulations by FDTD method have been carried out to demonstrate the effectiveness of the proposed method.

\section{The Novel Time Reversal Method for Target Detection}

Let us consider a medium where a significant number of highly reflective scatterers exist. Assume that we can probe the medium without the presence of a target by transmitting pulsed electromagnetic wave using an array of receivers/transmitters. The reflection of the transmitted wave from the scatterers received by each receiver/transmitter is recorded. Each recorded waveform is time reversed and re-transmitted back to the medium synchronously for all the receiver/transmitters. Now, the re-transmitted time reversed wave will focus, only, on the scatterers where the initial reflections occur.

Based on the above described time reversal phenomenon, our method can be described as the following: Instead of re-transmit the exactly time reversed signal, the time reversed signal at each receiver/transmitter is modified by a multiplication factor of a complex number such that the re-transmitted wave destructively interfere at each scatterer and little, or no, reflection would occur. In other words, the scatterers have been nullified and the highly scattering 
background has been effectively eliminated. Now, any appearance of additional reflective object, namely a target, will cause a reflection from the re-transmission of the modified time reversal wave.

In the situations that the scattering from cluttered medium could not be perfectly nullified, e.g. the number of scatterers is greater than the number of receiver/transmitters, the difference between the reflections of the re-transmission of the modified time reverse wave with and without the presence of the target is time reversed again and re-transmitted back to the medium. This iteration step results in a self-focusing of the re-transmitted wave on the target, effectively yielding a significant enhancement of the detection dynamic range as well as signal-to-noise ratio. The method, thus, is referred to as time reversal adaptive interference canceller (TRAIC) [5].

\section{The Multiplication Factors for the Modified Time Reversal Operation}

Assume that we have $\mathrm{N}$ number of receiver/transmitters in an antenna array and the echo received by the $i$-th receiver/transmitter after retransmitting the time reversed wave of the initial reflection, can be written as

$$
e_{i}(t)=\sum_{j=1}^{N} S_{i j}^{T R}(t)
$$

Where $S_{i j}^{T R}(t)$ denotes the reflection received by the i-th receiver/transmitter from the cluttered medium after retransmitting the time reversed initial reflection only by $\mathrm{j}$-th receiver/transmitter. Now we introduce the multiplication factors $\left\{A_{j}, j=1,2, \ldots, N\right\}$ such that the reflection received by the $\mathrm{i}$-th receiver/transmitter becomes

$$
n_{i}(t)=\sum_{j=1}^{N} A_{j} S_{i j}^{T R}(t)
$$

with the constraint

$$
\sum_{j=1}^{N} A_{j}^{*} A_{j}=N
$$

Let $\left\{n_{j}=0, j=1,2, \ldots, N\right\}$, i.e. perfectly nulling of all the scatterers in the medium, we have,

$$
\sum_{j=1}^{N} A_{j} S_{i j}^{T R}(t)=0
$$

If the number of scatterers is less than the number of receiver/transmitters, Eq.(4) will have nontrivial solutions. Otherwise, $\left\{A_{j}, j=1,2, \ldots, N\right\}$ can be obtained by minimizing $\left\{n_{j}, j=1,2, \ldots, N\right\}$. 


\section{Numerical Simulation Results and Discussion}

Finite-difference-time-domain (FDTD) method has been utilized to simulate the electromagnetic wave propagation in two-dimension (2D) for the purpose of demonstrating the TRAIC scheme. The field is descritized to square mesh cells with each cell size $1.2 \mathrm{~cm}$ on the side which is one-tenth of the wavelength. The calculation field is a $3.6 \mathrm{~m} \times 3.6 \mathrm{~m}$ area. A $12 \mathrm{~cm}$ thick perfectly matched layer (PML) is assumed for the all four side boundaries of the calculation field. Scatterers and the target are assumed to be perfect conductor. The frequency of the electromagnetic wave is chosen to be $\mathrm{f}=2.5 \mathrm{GHz}$. A linear array of six omnidirectional receiver/transmitter is used for performing receiving and retransmission. Each receiver/transmitter is a line source with electric field vector in the direction perpendicular to the plane of the $2 \mathrm{D}$ calculation field. Adjacent receivers/transmitters are separated by $24 \mathrm{~cm}$, two times of the wavelength.

Figure 1 shows the results of nullifying one scatterer. Initially, a $2.5 \mathrm{~ns}$ pulsed wave wave is synchronously transmitted by the receiver/transmitter array to the field with one scatterer. The reflection from the scatterer is received by the receiver/transmitters. Figure 1(a) shows the synchronous retransmission of the time reversed wave and the wave propagation focuses on the scatterer. Figure 1(b) shows the synchronous retransmission of the modified time reversed wave with a set of $\left\{A_{j}, j=1,2, \ldots, 6\right\}$ obtained by solving Eq. (4). This time, the wave propagation completely "avoids" the scatterer, yielding no return echo as shown in Fig. 1(c).

Figure 2 shows a situation of a medium with two scatterers. With the method described above, the two scatterers can be nullified using the modified time reversed received reflections. An echo will be received when the target becomes present. Performing the time reversal operation on the received echo and

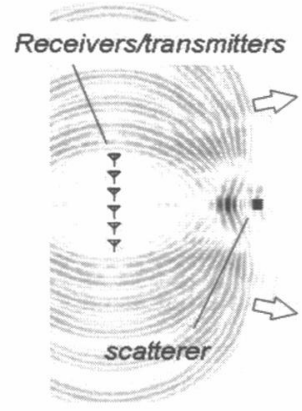

(a)

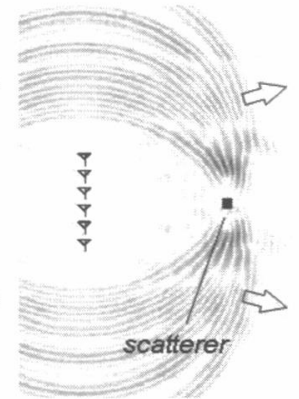

(b)

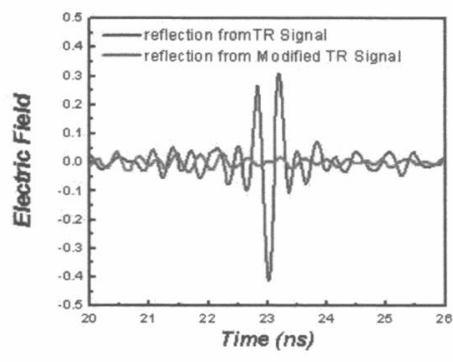

(c)

Fig. 1. (a) Retransmission of time reversed wave after the initial reflection and the wave propagation focuses on the scatterer. (b) Retransmission of the modified time reversed wave and note that the wave propagation completely misses the scatterer. The echo after the retransmission of the time reversed wave (blue) and the virtually no echo is received after the retransmission of the modified time reversed wave. 


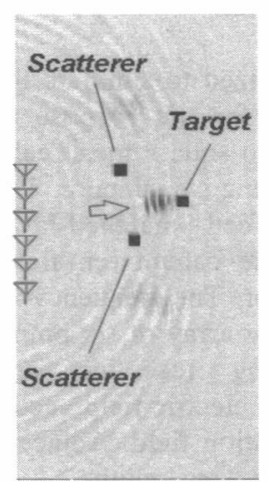

Fig. 2. Amplitude plot during the retransmission of the time reversed difference signal $w /$ and w/o target.

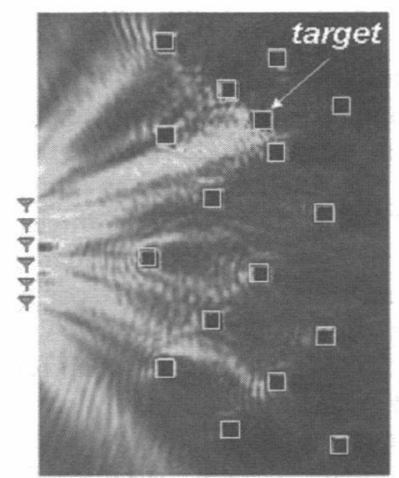

(a)

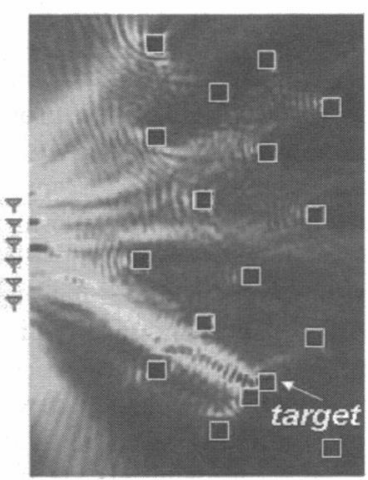

(b)

Fig. 3. Color-map of the magnitude trace of the Poynting vector during the retransmission of the time reversed difference signal. In each case, there are 16 scatterers in the field, one target. Self focusing on the target is evident.

retransmitting it back to the medium, yielding a strong focusing of the propagated wave on the target. Figure 3 shows two cases where the medium comprises 16 scatterers. The scatterers can not be perfectly nullified. In this case, the difference of the received reflections received after the retransmission of the modified time reversed wave with and without the target is time reversed again and retransmitted back to the medium. The self-focusing of the wave on the target is very evident.

\section{Conclusions}

We have developed a novel method for EM wave target detection in a cluttered medium by utilizing the concept of time reversal. Numerical simulation has shown that the method significantly enhances the dynamic range of the target detection in a highly scattering environment and could substantially increase target detectability.

\section{References:}

[1] M. Fink, "Time-reversed acoustics", Physics Today, vol. 50, pp. 34-40, 1997.

[2] H. C. Song, S. Kim, "Environmentally adaptive reverberation nulling using a time reversal mirror", Journal of Acoust. Soc. Am., vol. 116, August 2004

[3] G. Lerosey, "Time reversal of electromagnetic waves", Physical Review Letters, vol. 92, no. 19, May 2004.

[4] B. E. Henty and D. D. Stancil, "Multipath-enabled super-resolution for $\mathrm{RF} /$ microwave communication using phase-conjugate arrays," Phys. Rev. Lett., accepted for publication.

[5] J. Moura, Y.W. Jin, D. Stancil, J.-G. Zhu, A. G. Gepni, Y. Jiang, B. Henty, "Single Antenna Time Reversal Adaptive Interference Cancellation," ICASSP'05, Philadelphia, PA, March, 2005. 